\title{
Head motion in children with ADHD during resting-state brain imaging
}

Xiang-zhen Kong ${ }^{1,2}$

${ }^{I}$ State Key Laboratory of Cognitive Neuroscience and Learning \& IDG/McGovern Institute for Brain Research, Beijing Normal University, Beijing, 100875, China $;{ }^{2}$ Center for Collaboration and Innovation in Brain and Learning Sciences, Beijing Normal University, Beijing, 100875, China.

Correspondence: kongxiangzheng@gmail.com

\section{Abstract}

Although head motion during scanning has been largely considered to reflect simply technical artifacts, there is growing evidence showing that the variable of head motion reflects valuable information regarding individual's psychological and/or clinical factors. Detailed studies would not only help to deal with the head motion biases, but they also help researchers in understanding the mental disorders. In this study, children with ADHD and demographically-matched typically developing control (TDC) participants underwent rs-fMRI examination without any specific task, and six mean single head motion parameters (three translational and three rotational) and a summary motion index for each participant were obtained. We found that patients with ADHD showed specific patterns of head motion during scanning: motion was significantly increased in the ADHD group, which was mainly contributed by the motion around and along the superior-to-inferior direction. Furthermore, the cross-validation classification analyses showed that the head motion could accurately distinguish children with ADHD from the healthy controls. These results suggest that head motion during scanning reflects useful information about the participants and accounting for head motion from MRI data may be helpful for ADHD diagnosing and treatment with neuroimaging. 


\section{Introduction}

Head motion during MRI scan is undesirable in brain mapping studies, because it not only displaces the brain in space, but also interferes with the MR signals (Friston et al., 1996; Bullmore et al., 1999; Jenkinson et al., 2002; Jezzard et al., 1998; Tijssen et al., 2009). Moreover, several studies have suggested that in-scanner head motion leads to systematic biases in the analyses of functional connectivity based on resting-state fMRI (rs-fMRI) data (Power et al., 2012; Van Dijk et al., 2012; Satterthwaite et al., 2012). Thus, the head motion during scanning has been largely considered to reflect simply technical artifacts, which may even overthrow some leading theories on certain disorders (e.g., autism spectrum disorders) (Deen and Pelphrey 2012). However, there is growing evidence that individual differences in head motion may be an important variable in itself, rather than simply a confounding variable.

Actually, recent studies have shown that head motion is fairly consistent across MRI scans, with test-retest reliability estimates in the moderate range (around 0.60) (Van Dijk et al., 2012; Zeng et al., 2014; Kong 2014), suggesting that head motion may reflect a trait-like property of the participants (e.g., Yan et al., 2013). Furthermore, the issue has been investigated from the behavioral, neuroimaging, and genetic perspectives. Behaviorally, a recent study (Kong et al., 2014) has shown that individual differences in impulsivity predict head motion during scanning, providing the first empirical evidence that links in-scanner head motion to psychological traits. Similarly, a recent neuroimaging study (Zeng et al., 2014) has found that distant connectivity primarily in the default mode network significantly correlates with individuals' head motion during scanning, suggesting that head motion is an indicator of a specific cognitive control capacity in the individual brain. Genetically, head motion has also been shown to be moderately heritable (around 40\%), according to a recent twin study (Couvy-Duchesne et al., 2014). Taken together, these latest studies suggest that the variable of head motion reflects valuable information regarding individual's psychological and/or clinical factors (Kong et al., 2014; Pujol et al., 2014; Strikwerda-Brown et al., 2014).

Actually, many patients, particularly those with motor control difficulties, will move much more than the healthy controls during scanning, even without any task. Although this seems an obvious problem, it has been rarely investigated. In addition, only a handful of research focuses on task-related head motion on schizophrenics (Bullmore et al., 1999; Yoo et al., 2005; Mayer et al., 2007) and stroke patients (Seto et al., 2001). It still requires additional investigations about head motion during rs-fMRI scanning. Additionally, studies in other patient population are also needed. 
Attention-deficit/hyperactivity disorder (ADHD) is one of the most common neuropsychiatric disorders among childhood. It is conservatively estimated that $3 \%$ to $9 \%$ of school-aged children are suffering from this disease (Anderson et al., 1987; Bird et al., 1988; Szatmari et al., 1989). Brain imaging studies on ADHD typically compare a group of ADHD children with the disorder to a group of typically developing control (TDC) (for a recent review, see Liston et al., 2011). In addition, MRI data from patients with ADHD contained more volumes with excessive head motion (e.g., > 2 mm) (Durston et al., 2003; Kaiser et al., 2010), implying that they move more intensively than healthy controls. However, there is still no systematic research about in-scanner head motion comparisons between ADHD and TDC groups. For example, whether patients with ADHD show specific patterns of head motion during scanning, and whether and what extend head motion could be used to facilitate classification.

To address these issues, this report is aimed to show detailed comparisons of head motion parameters between children with and without ADHD under rs-fMRI examinations. First, we evaluated the head motion parameters of all children with rs-fMRI data and then quantitatively compared the results between the two groups. Further, we examined the predictability of ADHD on the basis of head motion during scanning.

\section{Methods}

\subsection{Participants and data acquisition}

The dataset used in this study was from the ADHD-200 Consortium (see ADHD-Consortium, 2012 and Brown et al., 2012). We used the data collected from Beijing to minimize the variability across institutions. There were 245 children, 143 of whom were TDC (59 females; mean age $=11.43 \pm 1.86$ years $)$, and the rest 102 were patients with ADHD (12 females; mean age $=12.08 \pm 2.04$ years). All participants (ADHD and TDC) were evaluated by the Schedule of Affective Disorders and Schizophrenia for Children-Present and Lifetime Version (KSADS-PL) with one parent for the establishment of the diagnosis.

The resting-state fMRI images were collected using a T2*-weighted gradient-echo EPI $\left(\right.$ GRE-EPI) sequence: $\mathrm{TR}=2 \mathrm{~s}, \mathrm{TE}=30 \mathrm{~ms}$, flip angle $=90^{\circ}, \mathrm{FOV}=220 \mathrm{~mm}$, matrix size $=64 \times$ 64, 30 axial slices, slice thickness $=4.5 \mathrm{~mm}$. Participants were instructed to keep their eyes closed and to relax during scanning. The total scan time lasted 8 min. Foam padding was used to restrict head motion within the scanner. In-scanner head motion was calculated from the resting-state fMRI images using the procedures described below. 
Other details about measurements and inclusion criteria are available on the website ADHD-200.

\subsection{In-scanner head motion calculation.}

In neuroimaging studies, it is a standard practice to estimate the position of the head at each volume and to realign all volumes using affine transformations. Head motion estimation involved these series of affine transformations, $T i$, where $i$ indexes volume and $T i$ spatially registers volume $i$ to a select reference volume (e.g., the first volume). Each transformation can be expressed as a combination of rotation and displacement components. Thus,

$$
T_{i}=\left[\begin{array}{cccc} 
& A_{i} & & t_{i} \\
0 & 0 & 0 & 1
\end{array}\right]
$$

where $\mathrm{Ai}$ is a $3 \times 3$ rotation matrix and it is a $3 \times 1$ column vector of translations. Ai can be factored into three elementary rotations (pitch, yaw, and roll) about each of the three axes.

Differentiating the transformations across contiguous volumes, yields $A$, a $3 \times 3$ rotation matrix, and $t$, a 3x1 column vector of displacements.

$$
T_{i}-T_{i-1}=T_{i} T_{i-1}^{-1}=\left[\begin{array}{cccc} 
& A & & t \\
0 & 0 & 0 & 1
\end{array}\right]
$$

Three rotation parameters and three displacement parameters can be evaluated with the method above. In practice, root mean squared (RMS) deviation, a summary statistic of in-scanner head motion, is widely used, since it summarizes six translations and rotations across all three axes (Jenkinson, 2002). The summary head motion RMS has been widely used in fMRI and DTI images processing to check the extent of head motion and make decisions about cohort formation or matching. It can be calculated directly from the affine matrices (Jenkinson, 1999). That is,

$$
\mathrm{RMS}=\sqrt{\frac{1}{5} R^{2} \operatorname{Trace}\left(A^{T} A\right)+t^{T} t}
$$

where RMS is the RMS deviation in $\mathrm{mm}, \mathrm{R}$ is a radius specifying the volume of interest $(\mathrm{R}=80$ $\mathrm{mm}$, approximately the mean distance from the cerebral cortex to the center of the head).

In this study, we obtained one affine transformation for each brain volume registering to the first volume using FLIRT from FSL (http://www.fmrib.ox.ac.uk/fsl/). First, we got the six translational and rotational parameters for each volume from each transformation using avscale from FSL. In addition, the summary head motion RMS was calculated from two transformations for two continuous volumes using rmsdiff from FSL. That is, the in-scanner head motion was measured as the displacement of each brain volume as compared to the previous volume, which is 
often used to test the head motion effects (e.g., Van Dijk et al., 2012;Satterthwaite et al., 2012). All the seven head motion parameters were averaged across all volumes for each participant.

Ten participants (6 ADHD and 4 TDC) showed large head movements (with the $1.5 * \mathrm{IQR}$ rule of the summary head motion RMS, $0.2 \mathrm{~mm}$ ) and thus were excluded from our analyses. In addition, 28 patients who had taken psycho-stimulant medications within 48 hours prior to scanning were also excluded to avoid the medicine effects. Our analyses were based on the data of the remaining 207 participants: 67 ADHD ( 9 female; mean age $=12.26 \pm 2.04$ years) and 140 TDC (59 female; mean age $=11.43 \pm 1.85$ years) .

\subsection{Prediction of ADHD with in-scanner head motion}

The predictability of ADHD on the basis of in-scanner head motion was examined. The summary head motion index and six single head motion parameters, along with gender and age, were used as the classification features, whereas children's statuses (i.e., ADHD or TDC) were used as the classification labels. Support Vector Machine (SVM) algorithm (insert reference) was used and validated with the leave-one-out cross-validation method. The statistical significance was computed by a permutation test: the probability distribution of correct classification was estimated by running the same SVM analysis on 1000 surrogate samples created by randomizing their labels. The significance of the prediction accuracy with the original data was then estimated as the probability that the original accuracy was exceeded by chance.

\section{Results}

\subsection{Overview of motion parameters within each group}

First, we examined the six head motion parameters within each group. The mean single head motion parameters were plotted in Fig. 1A, 1B. In terms of these parameters, the mean head motion parameters were much less than $1 \mathrm{~mm}$ for translations or $1^{\circ}$ for rotations during rs-fMRI data acquisition for both control and patient groups. This indicates that most of the participants were able to undergo the rest-state fMRI scanning without significant head motion.

We performed paired t-tests to compare the pairs of head motion parameters within each group. For translation parameters, motion along the $\mathrm{x}$-axis (left-to-right) was significantly less than motion along the $y$ - and z-axis for both groups (ADHD: $\mathrm{t}(66)>8.40, \mathrm{p}<0.001$, corrected with Bonferroni correction; TDC: $\mathrm{t}(139)>10.00, \mathrm{p}<0.001$, corrected). In terms of rotational parameters, the pitch rotation (around the $\mathrm{x}$-axis) was significantly greater than rotations about the two other axes for both groups (ADHD: $t(66)>3.50, \mathrm{p}<0.001$, corrected; TDC: $\mathrm{t}(139)>5.10, \mathrm{p}<$ 0.001 , corrected). There was no significant difference in other pairs of head motion parameters 
1 (ps > 0.15), except that for patients group, motion along z-axis (superior-to-inferior) was

2 significantly greater than motion along the $y$-axis $(t(66)=3.32, p=0.001$, corrected). In addition, the translations along the z-axis (superior-to-inferior) and pitch rotation were the most significant motion patterns for both control and patients groups.
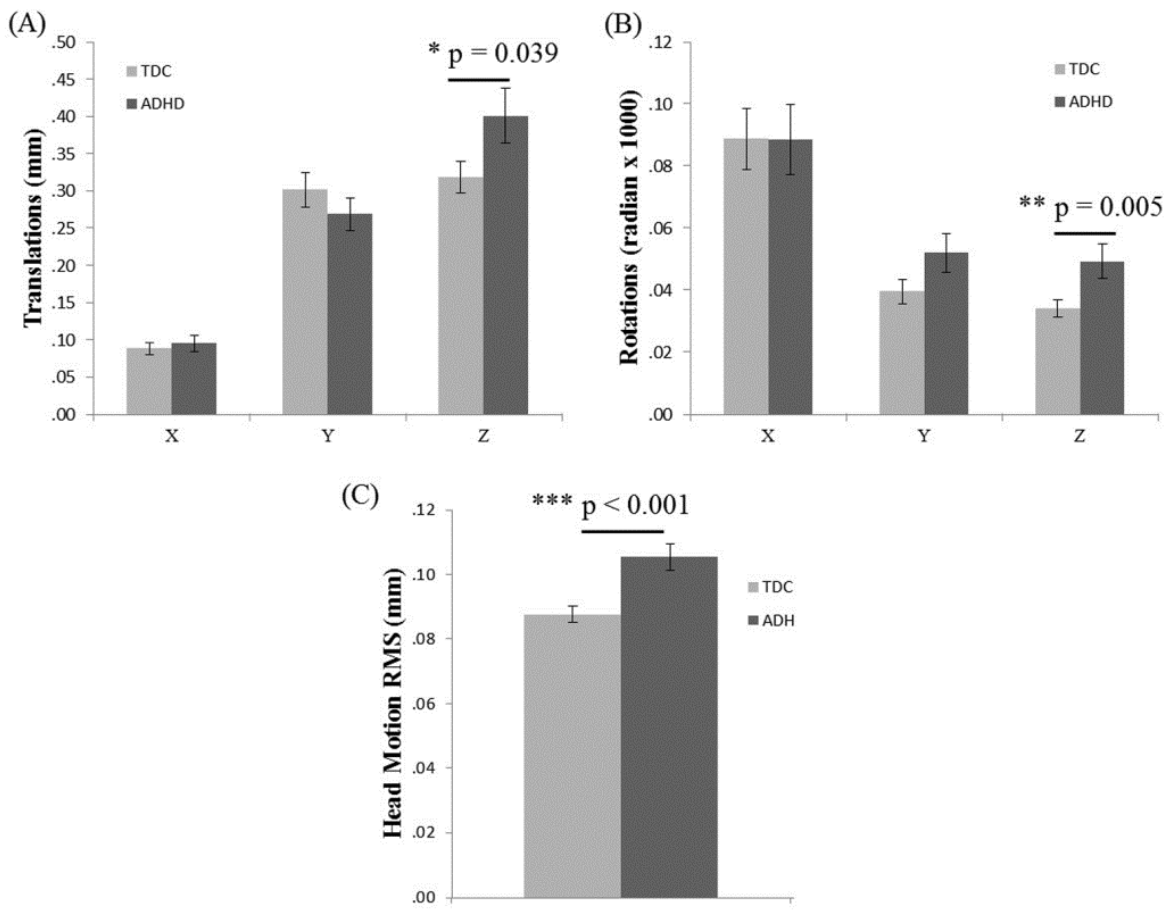

Fig. 1. Head motion patterns for the ADHD and TDC groups. In (A), three translation parameters for each group were shown. In (B), three rotation parameters for each group were shown. In $(\mathrm{C})$, the summary index of head motion was increased in the ADHD group compared to the TDC group. Error bars indicate \pm 1 SE. Asterisks indicate a significant difference between groups $(* \mathrm{p}<.05, * * \mathrm{p}<.01, * * * \mathrm{p}<.001)$

\subsection{Group comparison of motion parameters}

Independent-samples t-tests were applied to the six head motion parameters and the summary head motion RMS, comparing children with and without ADHD. In terms of the six parameters, we found that the yaw rotation (around the z-axis) was increased in ADHD group compared to that in the TDC group $(t(205)=2.82, p=0.005$, corrected; Fig. 1B). Similarly, we found that the motion along the z-axis also tended to increase in ADHD group compared to the TDC group $(\mathrm{t}(205)=2.07, \mathrm{p}=0.039$, uncorrected; Fig. 1A). We did not find any group difference in the remaining four motion parameters (all $\mathrm{p}>0.15$ ), only a marginal significant group difference in the roll rotation (around the $y$-axis; $p=0.069$, uncorrected). In terms of the summary RMS, we found that the motion was increased in ADHD group compared to the TDC group $(\mathrm{t}(205)=4.00, \mathrm{p}$ $<0.001$, corrected; Fig. 1C). This suggests that, though there was no 'sudden' bad head motion 
for both groups, children with ADHD actually showed greater head motion than the control group, particularly in terms of the rotation around z-axis and motion along z-axis.

\subsection{Prediction of ADHD with in-scanner head motion}

The leave-one-out across-validation showed that the average accuracy of the prediction of children's status was $71.5 \%$ ( $<<0.001$, permutation test). These results suggest that the head motion parameters during scanning could accurately distinguish children with ADHD from the healthy control.

\section{Discussion}

Although head motion during scanning has been largely considered to reflect simply technical artifacts, there is growing evidence showing that the variable of head motion reflects valuable information regarding individual's psychological and/or clinical factors (Kong et al., 2014; Pujol et al., 2014; Cherie et al., 2014). Detailed studies may not only help to deal with the head motion biases, but they also help researchers in understanding the mental disorders. In this study, we found that patients with ADHD showed specific patterns of head motion during scanning. Furthermore, the cross-validation classification analyses showed that the head motion could accurately distinguish children with ADHD from the healthy controls.

To date, few studies have investigated the specific patterns of head motion in patients with ADHD. One early study with task fMRI reported increased head motion in ADHD compared with TDC (Epstein et al., 2007). However, they did not find any significant difference, which might be due to the small sample size $(n=12)$. Moreover, they did not examine the group difference in head motion along/around single directions, which may be more important for prevention of head motion during imaging children with ADHD. In our present study, we found that in both groups, motion along the $\mathrm{x}$-axis (left-to-right) was the smallest in those along the three axes and the pitch rotation (around the $\mathrm{x}$-axis) was the greatest in those three rotations. Specially, for ADHD group, motion along z-axis (superior-to-inferior) was significantly greater than those along other two axes. Furthermore, to test the hypothesis that children with ADHD move more than TDC population, we conducted independent-samples t-tests on single motion parameters. As expected, we found a significant increase on the motion in patient group compared to the control group. And the increase was mainly due to the yaw rotation (around the z-axis) and motion along z-axis (superior-to-inferior). More importantly, the final classification analysis showed that the head motion parameters during scanning could accurately distinguish children 
with ADHD from the healthy control group. These suggest that taking the head motion information from MRI data into account is expected to improve clinical diagnosis and evaluation of treatment for patient with ADHD and even other disorders.

An interesting aspect from the data is that the increase of head motion in the ADHD group was mainly contributed by the motion along and around z-axis, rather than other four motion parameters. In the view of reducing head motion biases, a mock scanner compliance training protocol (e.g., Seto et al., 2001; Epstein et al., 2007) or passive head restraints (e.g., Green et al., 1994; Menon et al., 1997) specific to these two kinds of motion may largely reduce the group difference. Additionally, move much more around/along z-axis may be a behavioral marker for ADHD diagnosing and treatment. Further studies are needed to examine the different head motion patterns for each subtype of ADHD, such as ADHD-Combined, ADHD-Hyperactive/Impulsive, and ADHD-Inattentive (Barkley, 1997).

There are some limitations in our study. First, the head motion was measured indirectly using registration of volumes. In the future studies, using actometers or infrared CCD-based methods may give more direct measurement. Second, in consideration of the coupling effect of head motion in the fMRI signals, we didn't include fMRI metrics in this study. Using independent scanning runs to get the fMRI metrics and head motion may be a possible approach to explore the neural basis of head motion.

Nevertheless, our study, for the first time, quantitatively showed the in-scanner head motion characteristics and the group differences between children with and without ADHD using rs-fMRI. We demonstrated that children with ADHD had different head motion patterns compared with TDC children. In this way, when using local and global brain neuroimaging parameters, taking head motion into account is expected to improve clinical diagnosis and evaluation of treatment for children with ADHD, and to have wider applications in diagnosis of other mental disorders. In addition, head motion behaves like a psychological trait and this possibility may be carefully considered in genetic and heritability analyses. 
1 Acknowledgments

2 We thank the ADHD-200 projects for data acquisition and sharing.

3 
References

Aksoy M, Liu C, Moseley ME, and Bammer R. 2008. Single-step nonlinear diffusion tensor estimation in the presence of microscopic and macroscopic motion. Magn Reson Med 59:1138-1150.

Anderson JC, Williams S, McGee R, and Silva PA. 1987. DSM-III disorders in preadolescent children. Prevalence in a large sample from the general population. Arch Gen Psychiatry 44:69-76.

Barkley RA. 1997. Behavioral inhibition, sustained attention, and executive functions: constructing a unifying theory of ADHD. Psychol Bull 121:65-94.

Bird HR, Canino G, Rubio-Stipec M, Gould MS, Ribera J, Sesman M, Woodbury M, Huertas-Goldman S, Pagan A, and Sanchez-Lacay A. 1988. Estimates of the prevalence of childhood maladjustment in a community survey in Puerto Rico. The use of combined measures. Arch Gen Psychiatry 45:1120-1126.

Brown MR, Sidhu GS, Greiner R, Asgarian N, Bastani M, Silverstone PH, Greenshaw AJ, and Dursun SM. 2012. ADHD-200 Global Competition: diagnosing ADHD using personal characteristic data can outperform resting state fMRI measurements. Front Syst Neurosci 6:69.

Bullmore ET, Brammer MJ, Rabe-Hesketh S, Curtis VA, Morris RG, Williams SC, Sharma T, and McGuire PK. 1999. Methods for diagnosis and treatment of stimulus-correlated motion in generic brain activation studies using fMRI. Hum Brain Mapp 7:38-48.

Chen B, Guo H, and Song AW. 2006. Correction for direction-dependent distortions in diffusion tensor imaging using matched magnetic field maps. Neuroimage 30:121-129.

Couvy-Duchesne B, Blokland GA, Hickie IB, Thompson PM, Martin NG, de Zubicaray GI, McMahon KL, and Wright MJ. 2014. Heritability of head motion during resting state functional MRI in 462 healthy twins. Neuroimage 102P2:424-434.

Deen B, and Pelphrey K. 2012. Perspective: Brain scans need a rethink. Nature 491:S20.

Durston S, Tottenham NT, Thomas KM, Davidson MC, Eigsti I-M, Yang Y, Ulug AM, and Casey BJ. 2003. Differential patterns of striatal activation in young children with and without ADHD. Biol Psychiatry 53:871-878.

Epstein JN, Casey BJ, Tonev ST, Davidson M, Reiss AL, Garrett A, Hinshaw SP, Greenhill LL, Vitolo A, Kotler LA, Jarrett MA, and Spicer J. 2007. Assessment and prevention of head motion during imaging of patients with attention deficit hyperactivity disorder. Psychiatry Res 155:75-82.

Friston KJ, Williams S, Howard R, Frackowiak RS, and Turner R. 1996. Movement-related effects in fMRI time-series. Magn Reson Med 35:346-355. 
Green MV, Seidel J, Stein SD, Tedder TE, Kempner KM, Kertzman C, and Zeffiro TA. 1994.

Head movement in normal subjects during simulated PET brain imaging with and without head restraint. J Nucl Med 35:1538-1546.

Jenkinson M, Bannister P, Brady M, and Smith S. 2002. Improved optimization for the robust and accurate linear registration and motion correction of brain images. Neuroimage 17:825-841.

Jezzard P, Barnett AS, and Pierpaoli C. 1998. Characterization of and correction for eddy current artifacts in echo planar diffusion imaging. Magn Reson Med 39:801-812.

Kaiser MD, Hudac CM, Shultz S, Lee SM, Cheung C, Berken AM, Deen B, Pitskel NB, Sugrue DR, Voos AC, Saulnier CA, Ventola P, Wolf JM, Klin A, Vander Wyk BC, and Pelphrey KA. 2010. Neural signatures of autism. Proc Natl Acad Sci U S A 107:21223-21228.

Kong XZ. 2014. Association between in-scanner head motion with cerebral white matter microstructure: a multiband diffusion-weighted MRI study. PeerJ 2:e366.

Kong XZ, Zhen Z, Li X, Lu HH, Wang R, Liu L, He Y, Zang Y, and Liu J. 2014. Individual differences in impulsivity predict head motion during magnetic resonance imaging. PLoS One 9:e104989.

Liston C, Malter Cohen M, Teslovich T, Levenson D, and Casey BJ. 2011. Atypical prefrontal connectivity in attention-deficit/hyperactivity disorder: pathway to disease or pathological end point? Biol Psychiatry 69:1168-1177.

Mayer AR, Franco AR, Ling J, and Canive JM. 2007. Assessment and quantification of head motion in neuropsychiatric functional imaging research as applied to schizophrenia. J Int Neuropsychol Soc 13:839-845.

Menon V, Lim KO, Anderson JH, and Pfefferbaum A. 1997. A head coil bite bar for reducing movement related artifacts during functional MRI scanning. Behavior Research Methods, Instruments, \& Computers 29:589-594.

Power JD, Barnes KA, Snyder AZ, Schlaggar BL, and Petersen SE. 2012. Spurious but systematic correlations in functional connectivity MRI networks arise from subject motion. Neuroimage 59:2142-2154.

Pujol J, Macia D, Blanco-Hinojo L, Martinez-Vilavella G, Sunyer J, de la Torre R, Caixas A, Martin-Santos R, Deus J, and Harrison BJ. 2014. Does motion-related brain functional connectivity reflect both artifacts and genuine neural activity? Neuroimage 101:87-95.

Reese TG, Heid O, Weisskoff RM, and Wedeen VJ. 2003. Reduction of eddy-current-induced distortion in diffusion MRI using a twice-refocused spin echo. Magn Reson Med 49:177-182. 
Rohde GK, Barnett AS, Basser PJ, Marenco S, and Pierpaoli C. 2004. Comprehensive approach for correction of motion and distortion in diffusion-weighted MRI. Magn Reson Med 51:103-114.

Satterthwaite TD, Wolf DH, Loughead J, Ruparel K, Elliott MA, Hakonarson H, Gur RC, and Gur RE. 2012. Impact of in-scanner head motion on multiple measures of functional connectivity: relevance for studies of neurodevelopment in youth. Neuroimage 60:623-632.

Seto E, Sela G, McIlroy WE, Black SE, Staines WR, Bronskill MJ, McIntosh AR, and Graham SJ. 2001. Quantifying head motion associated with motor tasks used in fMRI. Neuroimage 14:284-297.

Strikwerda-Brown C, Davey CG, Whittle S, Allen NB, Byrne ML, Schwartz OS, Simmons JG, Dwyer D, and Harrison BJ. 2014. Mapping the relationship between subgenual cingulate cortex functional connectivity and depressive symptoms across adolescence. Soc Cogn Affect Neurosci.

Szatmari P, Offord DR, and Boyle MH. 1989. Ontario Child Health Study: prevalence of attention deficit disorder with hyperactivity. J Child Psychol Psychiatry 30:219-230.

The ADHD-200 Consortium. 2012. The ADHD-200 Consortium: A Model to Advance the Translational Potential of Neuroimaging in Clinical Neuroscience. Front Syst Neurosci 6:62.

Tijssen RH, Jansen JF, and Backes WH. 2009. Assessing and minimizing the effects of noise and motion in clinical DTI at 3 T. Hum Brain Mapp 30:2641-2655.

Van Dijk KR, Sabuncu MR, and Buckner RL. 2012. The influence of head motion on intrinsic functional connectivity MRI. Neuroimage 59:431-438.

Yan CG, Cheung B, Kelly C, Colcombe S, Craddock RC, Di Martino A, Li Q, Zuo XN, Castellanos FX, and Milham MP. 2013. A comprehensive assessment of regional variation in the impact of head micromovements on functional connectomics. Neuroimage 76:183-201.

Yoo SS, Choi BG, Juh R, Pae CU, and Lee CU. 2005. Head motion analysis during cognitive fMRI examination: application in patients with schizophrenia. Neurosci Res 53:84-90.

Zeng LL, Wang D, Fox MD, Sabuncu M, Hu D, Ge M, Buckner RL, and Liu H. 2014. Neurobiological basis of head motion in brain imaging. Proc Natl Acad Sci U S A. 